\title{
Comparison of Reward Modulation in the Frontal Eye Field and Caudate of the Macaque
}

\author{
Long Ding and Okihide Hikosaka \\ Laboratory of Sensorimotor Research, National Eye Institute, National Institutes of Health, Bethesda, Maryland 20892
}

\begin{abstract}
The frontal eye field (FEF) influences saccade generation via direct projections to the superior colliculus and an indirect pathway through the basal ganglia. To test whether different reward information is represented in the FEF and the basal ganglia, we recorded from the FEF and the caudate nucleus in monkeys performing an asymmetrically rewarded memory-guided saccade task. A peripheral cue at one of two opposing positions was flashed briefly to indicate the saccade target. In a given block, one position was associated with big reward and the other with small reward. Big-reward position was alternated between blocks. In this task, the FEF and caudate displayed similar prevalence of neuronal activity before cue onset that was larger in blocks with specific big reward-cue position associations. They also exhibit comparable reward modulation of visual responses that were spatially selective. In contrast, visual responses that were specific to expected reward size, instead of spatial locations, were commonly observed in caudate but rarely seen in the FEF. Thus, both the FEF and basal ganglia may contribute to reward bias in saccade generation, with the FEF providing spatially relevant reward information and the basal ganglia providing additional reward-specific information.
\end{abstract}

Key words: cortex; basal ganglia; striatum; saccade; nonhuman primate; asymmetric reward

\section{Introduction}

Reward is a powerful modulator of behavior. To elucidate how expectation of reward is transformed into behavioral differences, it is important to know how different aspects of reward information are represented in different brain regions involved in the same behavioral task. The neural system underlying saccade generation includes two key players: the frontal eye field (FEF) and the superior colliculus (SC). FEF participates in saccade generation via direct projections to $\mathrm{SC}$ and an indirect pathway through the basal ganglia (Huerta et al., 1986; Stanton et al., 1988; Shook et al., 1991; Parthasarathy et al., 1992). Recently, it has been shown that SC and the basal ganglia [caudate and substantia nigra pars reticulata $(\mathrm{SNr})]$ contain substantial number of neurons with reward modulation (Kawagoe et al., 1998, 2004; Kobayashi et al., 2002; Lauwereyns et al., 2002a,b; Sato and Hikosaka, 2002; Takikawa et al., 2002; Ikeda and Hikosaka, 2003). In contrast, in different saccade tasks with manipulations of reward magnitude, FEF has been shown to contain few neurons with reward modulation (Leon and Shadlen, 1999; Coe et al., 2002; Roesch and Olson, 2003). These results led to the general hypothesis that FEF mainly encodes spatial information necessary for

Received Feb. 23, 2006; revised April 28, 2006; accepted May 15, 2006.

This work was supported by the Intramural Research Program of the National Institutes of Health-National Eye Institute. We thank Drs. Kae Nakamura and Masaki Isoda for valuable discussions and support. We are grateful to Drs. Kirk Thompson, Barry Richmond, Robert Wurtz, and two anonymous reviewers for their helpful comments on this manuscript. We thank our colleagues in the Laboratory of Sensorimotor Research for suggestions and the support staff for indispensable assistance.

Correspondence should be addressed to Long Ding, Laboratory of Sensorimotor Research, National Eye Institute, National Institutes of Health, Building 49, Room 2A50, Bethesda, MD 20892. E-mail: dingl@nei.nih.gov.

DOI:10.1523/JNEUROSCI.0836-06.2006

Copyright $\odot 2006$ Society for Neuroscience $\quad$ 0270-6474/06/266695-09\$15.00/0 saccade generation, whereas the basal ganglia provide additional motivational information.

In this study, we compared single neurons in both FEF and caudate nucleus in two monkeys performing an asymmetrically rewarded saccade task (see Fig. 1). We report here that spatially selective reward modulation was also observed in FEF at qualitatively comparable task periods and with similar prevalence to that seen in caudate. However, caudate exhibited additional spatially nonselective reward size modulation, which was extremely rare in FEF.

\section{Materials and Methods}

Two male rhesus monkeys (Macaca mulatta; monkey L, $5.3 \mathrm{~kg}$; monkey $\mathrm{D}, 7.3 \mathrm{~kg}$ ) were trained and used for electrophysiological recordings. All animal care and experimental procedures were approved by the Institute Animal Care and Use Committee and complied with the Public Health Service Policy on the humane care and use of laboratory animals.

Surgical procedures. Monkeys were anesthetized initially with intramuscular injections of ketamine $\mathrm{HCl}(10 \mathrm{mg} / \mathrm{kg})$, diazepam $(1 \mathrm{mg} / \mathrm{kg})$, and glycopyrrolate $(0.01 \mathrm{mg} / \mathrm{kg})$ and then maintained at a general anesthetized state with isofluorane. After the skull was exposed, acrylic screws were installed to fasten the dental acrylic head implant with the skull. Plastic head holders and recording chambers were placed stereotaxically and secured with dental acrylic. Rectangular recording chambers were positioned to gain access to both the frontal eye field and the caudate. Bilateral eye coils were implanted subconjunctivally using the methods described by Judge et al. (1980). Magnetic resonance (MR) images were taken in sagittal and coronal planes [monkey D, Philips (Andover, MA), 3 tesla; monkey L, GE (Waukesha, WI) Signa 2, 1.5 tesla]. Craniotomy was performed 4-6 months later, when monkeys were deemed well trained for the task and ready for electrophysiological recordings.

Behavioral tasks. Behavioral tasks were under the control of a QNXbased real-time experimentation data acquisition system [REX; Laboratory of Sensorimotor Research, National Eye Institute, National Insti- 
tutes of Health (LSR/NEI/NIH), Bethesda, MD]. Visual stimuli were rear-projected by an active matrix liquid crystal display projector onto a frontoparallel screen $25 \mathrm{~cm}$ from the monkey's eyes. Water reward was delivered through a spigot under the control of a solenoid valve. Monkeys were initially trained to perform a memory-guided saccade task (see Fig. 1). Briefly, the appearance of a central fixation point (diameter, $0.6^{\circ}$ ) signaled the trial initiation. Monkeys were required to maintain fixation within a window of $\sim 3.5^{\circ}$ for $2.1 \mathrm{~s}$. One second after monkeys began fixating, a $100 \mathrm{~ms}$ cue was flashed at one of two opposing positions. At the end of the fixation period, the fixation point was turned off and monkeys were required to make a saccade toward the previously cued position. Saccades that were initiated within $500 \mathrm{~ms}$ after the disappearance of the fixation point and landed in the target window within $150 \mathrm{~ms}$ after saccade onset were considered correct. The size of the target window was $3-11^{\circ}$, depending on the target eccentricity and location. Correct saccades were signaled by the reappearance of the peripheral cue with a 150 $\mathrm{ms}$ delay. Water reward followed with an additional $300 \mathrm{~ms}$ delay. Error trials were indicated with an auditory click and repeated until a correct response occurred.

After monkeys learned the basic memory-guided saccade tasks, asymmetric reward schedule was introduced. The asymmetrically rewarded memory-guided saccade tasks are modified from Kawagoe et al. (1998). Briefly, trials were run in blocks. In a given block, correct saccades to one target position was associated with large reward $(0.3-0.4 \mathrm{ml})$, whereas correct saccades to the other target position was associated with small reward $(0.075-0.1 \mathrm{ml})$. Each block consisted of $15-35$ correct trials, with random intertrial intervals in the range of $1-2.5 \mathrm{~s}$. The reward contingency was constant within a block and alternated between blocks. The beginning of a new block was signaled by a prolonged intertrial interval of $>5 \mathrm{~s}$. The selection of target position was pseudorandom and balanced using sub-blocks of six trials.

Recording. Eye movement was monitored using a scleral search coil system with $1 \mathrm{~ms}$ resolution. Single-unit recordings were performed using tungsten and platinum-iridium electrodes (Frederick Haer Company, Bowdoinham, ME). Single units were isolated on-line using a custom voltage-time window discrimination software (MEX; LSR/NEI/ NIH). FEF was identified as the anterior bank of the arcuate sulcus with low microstimulation threshold for evoking saccades. Caudate was identified by its characteristic low background activity and with the aid of MR images. Putative projection neurons in the caudate were identified by their low spontaneous activity and characteristic activation in bursts of spikes. All cells that showed task-related activation were recorded. Once a task-related neuron was isolated, cues at $45^{\circ}$ apart were used to estimate the near-optimal cue location. The near-optimal cue location and its opposite location across the fixation point were used for data collection.

To minimize differences in behavior between FEF and caudate recordings, in monkey L, recordings were made from FEF and caudate in alternate weeks in the first 2 months. The remaining recordings were completed in FEF first and then caudate. In monkey D, which had shown more stable behavioral bias at the beginning of this study, recordings were done in FEF first and then in caudate.

Data analysis. For analysis, only correct trials were included. Saccade onset latency was determined using standard velocity and acceleration threshold-crossing algorithms. The difference in mean saccade latency between small-reward trials and big-reward trials was used to ensure consistent and comparable motivation across neural recordings in FEF and caudate.

We analyzed neural spike time data aligned to cue onset and to saccade onset. For each neuron, spike density functions were constructed for each trial with a Gaussian kernel $(\sigma=20 \mathrm{~ms}$; width, $3 \sigma)$. These parameters were chosen as a compromise between temporal resolution and reliability. Qualitatively similar results were obtained using $\sigma=10 \mathrm{~ms}$. Spike density functions were grouped into four conditions with combinations of reward size and cue position. The remaining analyses were performed on these smoothed spike density functions for every $1 \mathrm{~ms}$ time bin. We performed two-way ANOVA analysis using reward size and cue position as the factors. With correction for multiple comparisons of the $35001 \mathrm{~ms}$ time bins (2500 and 1000 for alignments on cue onset and saccade onset, respectively), we defined statistical significance as $p<$
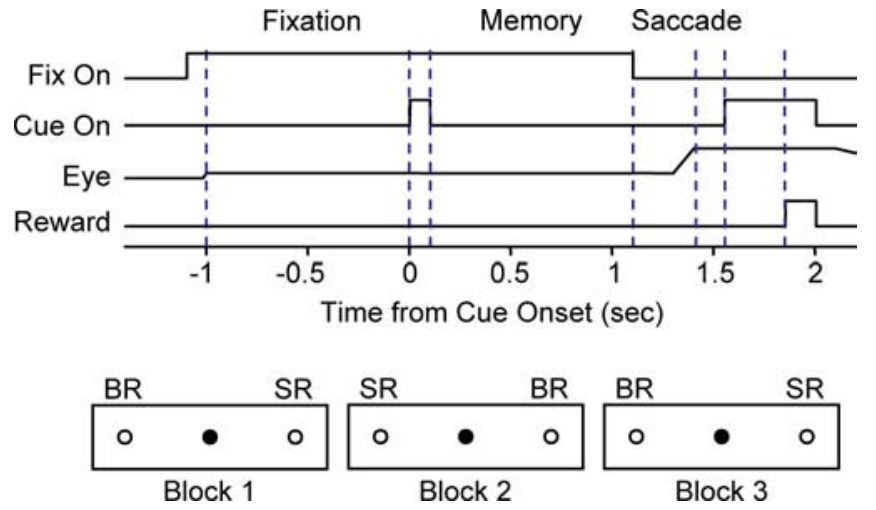

Figure 1. Asymmetrically rewarded memory-guided saccade task. The monkey maintains fixation on a central point (filled circle) for $2.1 \mathrm{~s}$. One second into the fixation period, a cue was flashed at one of two peripheral positions (open circles). After the fixation point was extinguished, the monkey was required to make saccade to the previously cued position to receive water reward. In a given block of trials, one position was associated with big reward (BR) and the other position was associated with small reward (SR). The reward position was reversed in the next block. A prolonged intertrial interval signaled a block change to the monkey.

$1.43 e^{-5}$, equivalent to a $p$ value of 0.05 . Time bins with significant results in ANOVA were examined further using Wilcoxon's rank sum tests.

We focused on four types of reward modulation in activity. (1) "Reward modulation in one cue position" indicates that the activity was primarily cue position selective and showed additional reward modulation in one cue position. "Cue position selective" indicates significant cue position main effect alone or, in case of concurrent significant interaction effect, significant difference between cue positions in both reward conditions and with the same sign. Wilcoxon's rank sum tests were performed to test for additional reward modulation for time bins with significant cue position selectivity. Significant reward modulation in either cue position qualified as "reward modulation in one cue position." (2) "Reward size selective" indicates significant reward size main effect alone or, in case of concurrent significant interaction effect, significant difference between reward conditions in both cue positions and with the same sign. (3) "Reward position selective" indicates significant interaction effect and significant difference between blocks (reward positions). (4) "Single condition selective" indicates significant interaction effect, significantly more/less activation in one condition, and no difference between the other three conditions. With these definitions, the second type of reward modulation was deemed not spatially selective, whereas the other types of reward modulation were spatially selective.

For each type of modulation, we generated a significance map for the whole-cell population. Each row of pixels represents data from a single cell. A black pixel indicates significant modulation at a given $1 \mathrm{~ms}$ time bin. For each time bin in the map, we also calculated the percentage of cells with significant modulation and estimated the $95 \%$ confidence intervals using bootstrap methods with 1000 iterations. To compare between FEF and caudate, we consider time bins with nonoverlapping confidence intervals as significantly different between the two populations.

To estimate the location of each neuron in the brain, we noted the coordinates of the electrode in the recording chamber and the depth of electrode at the time of recording. Using MR slices showing the edges of the recording chamber, we approximated the trajectory of the electrode penetration as perpendicular to the top surface of the recording chamber and estimated the neuron location by the electrode depth along the trajectory.

\section{Results}

Behavioral bias toward big reward is significant and comparable for FEF and caudate recordings

During recordings, monkeys performed an asymmetrically rewarded memory-guided saccade task (Fig. 1). Consistent with previous results (Kawagoe et al., 1998; Takikawa et al., 2002), 
A

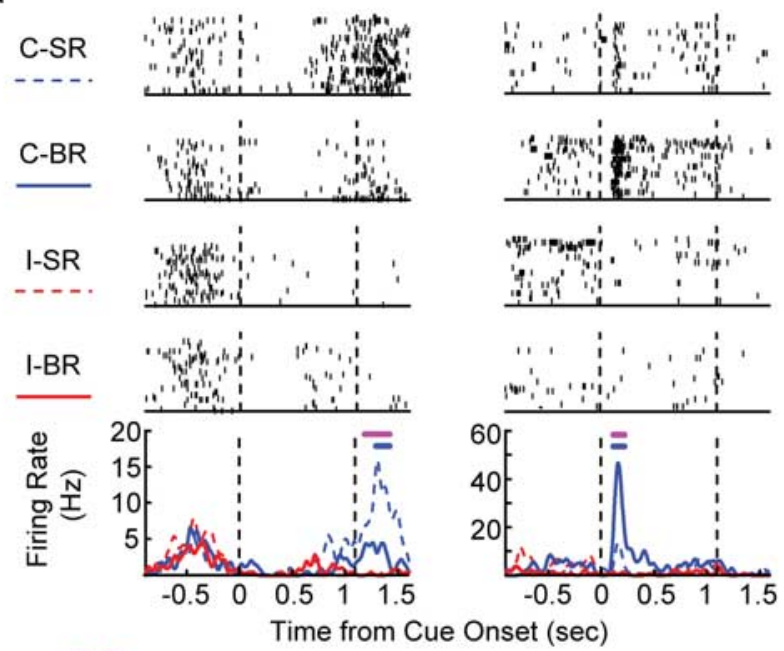

C
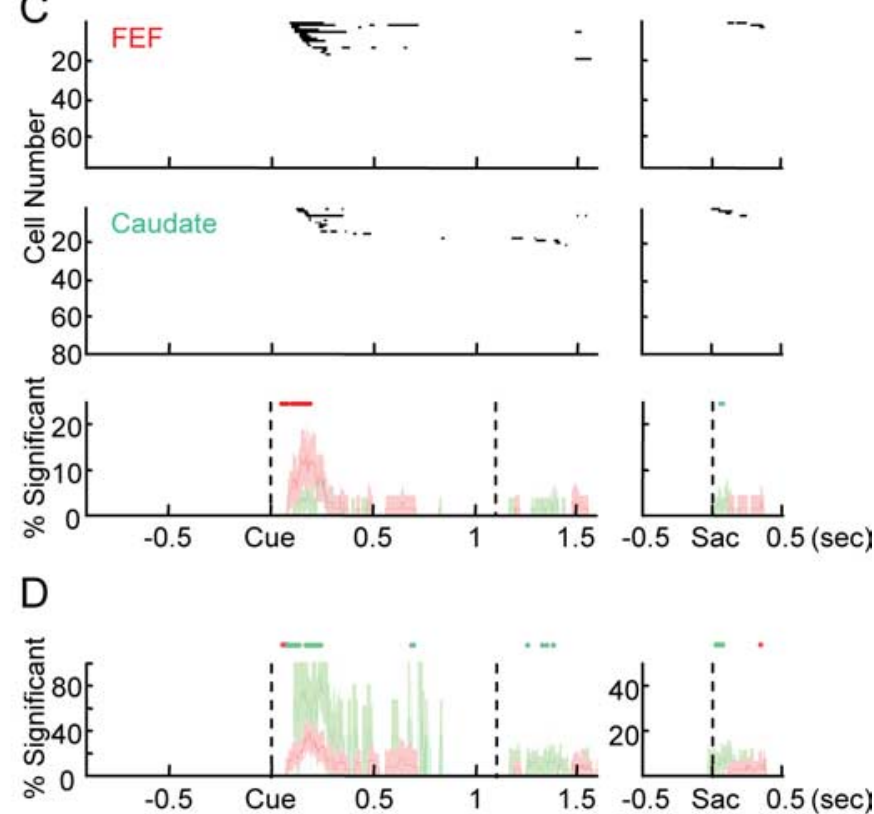

E
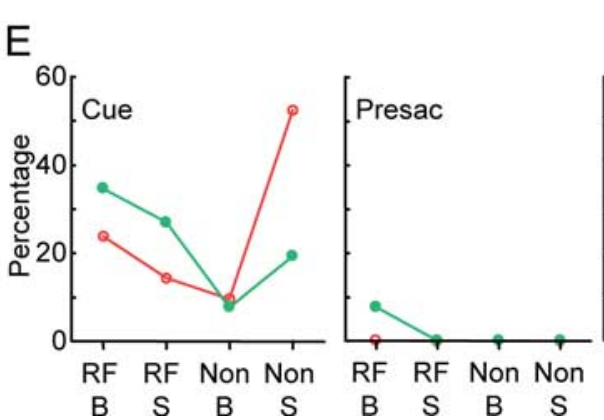

$\rightarrow$ FEF

$\rightarrow$ Caudate

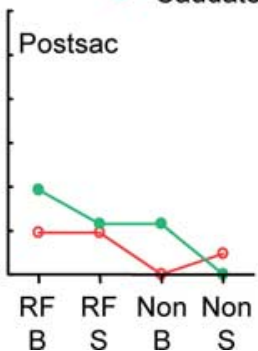

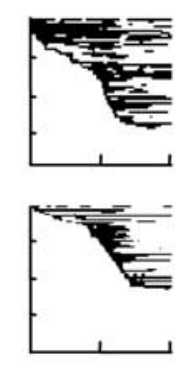

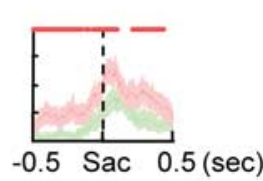

Figure 2. Cue position selectivity and reward modulation in one cue position. $A$, Examples of neural activity showing cue position selectivity and reward modulation in one cue position. Rasters were shown for the four conditions in the following order: contralateral cue-small reward (C-SR), contralateral cue- big reward (C-BR), ipsilateral cue-small reward (I-SR), and ipsilateral cue- big reward (I-BR). Dashed lines indicate cue onset and "go" signal, respectively. The bottom panel shows the spike density functions for all four conditions. Magenta bars indicate periods of response with significant cue position selectivity. Blue bars indicate periods of response with significant reward modulation for the contralateral cue position. $\boldsymbol{B}$, Population data for cue position selectivity. The top panel shows the significance maps for FEF neurons $(n=75)$ aligned to cue onset and saccade onset, respectively. The middle panel shows the significance maps for caudate neurons $(n=79)$. Black pixels indicate the time bins with statistically significant modulation. Data from a single cell are presented as a row of pixels. Cells are sorted according to the earliest time of significant modulation. The bottom panel shows the percentage of cells with significant modulation at every $1 \mathrm{~ms}$ time bin. Red, FEF; green, caudate. Lightly shaded areas are estimates of $95 \%$ confidence intervals. Red dots on top indicate time bins with significantly greater percentage for FEF than for caudate. $\boldsymbol{C}$, Population data for reward modulation in one cue position. The layout is the same as $\boldsymbol{B}$. Red and green dots indicate time bins with significantly greater percentage for FEF and for caudate, respectively. $\boldsymbol{D}$, Percentage of cells with significant reward modulation in one cue position, normalized by the number of cells with significant cue position selectivity. Red and green dots indicate time bins with significantly greater percentage for $F E F$ and for caudate, respectively. $E$, Frequency of occurrence for four types of reward modulation in one cue position. RF, Preferred cue location; Non, nonpreferred cue location; B, larger activation in big-reward trials; $S$, larger activation in small-reward trials; Cue, $0-1$ $s$ after cue onset; Presac, $0.25-0$ s before saccade onset; Postsac, $0-0.5$ s after saccade onset.

saccade latency and velocity were modulated by reward expectation. Both monkeys showed significant longer saccade latency in small-reward trials than in big-reward trials in both FEF and caudate recordings $(p<0.0001$, Wilcoxon's signed rank test). Furthermore, the difference in saccade latency between reward conditions was comparable between FEF and caudate recordings in both monkeys ( $p=0.1767$ and 0.3045 for monkeys $\mathrm{D}$ and $\mathrm{L}$, respectively, Mann-Whitney test). Both monkeys also showed significant higher peak velocity in big-reward trials $(p<0.0001$ and 0.01 for monkeys D and L, respectively, Wilcoxon's signed rank test). The difference in mean peak velocity between reward conditions was comparable between FEF and caudate recordings in both monkeys ( $p=0.7195$ and 0.3817 for monkeys $\mathrm{D}$ and $\mathrm{L}$, respectively, Mann-Whitney test). Thus, behavioral reward bias was significant and comparable between FEF and caudate recordings in both monkeys.
We recorded 75 neurons in FEF and 79 putative projection neurons in the caudate. All cells showed task-related activation in relation to various events during the task. In the next four sections, we will describe and compare, in FEF and caudate, representation of four types of reward modulation as defined in Materials and Methods.

Reward modulation in one cue position is present in both FEF and caudate

Examples of cue position-selective activity are shown in Figure $2 A$. The caudate neuron showed nonspecific activation during the fixation period before cue onset, low activity in the early memory period, and contralateral-selective activation in the late memory and the saccade periods. In the later part of the cue position-selective period (indicated by the magenta bar), the activation was larger in small-reward trials than in big-reward trials. 
The FEF neuron showed clear contralateral-selective activation in response to cue onset. This activation was larger in big-reward trials.

To summarize population data for each type of modulation, we generated binary significance maps as shown in the top panels in Figure 2, $B$ and $C$. Figure $2 B$ shows the population results for cue position-selective activities. In the FEF maps, dark pixels can be observed in almost all rows, indicating that most cells show cue position-selective activity. Furthermore, in the FEF map aligned to cue onset, $\sim 80 \%$ of the cells showed significant modulation in the memory period, in clear contrast to the $\sim 30 \%$ in the caudate map. To validate these observations, we calculated the percentage of cells showing significant modulation in every time bin, as shown in the bottom panel of Figure $2 B$, along with estimates of 95\% confidence intervals. Consistent with our first impression, the percentage of cue position-selective FEF cells stayed higher than that of caudate cells after cue onset. We considered nonoverlapping confidence intervals as indication of significant difference between FEF and caudate. Using this criterion, FEF showed significantly higher percentage of cue position-selective neurons $\sim 70 \mathrm{~ms}$ after cue onset and throughout the memory period. When aligned on saccade onset, FEF also showed a higher percentage mostly throughout the $1 \mathrm{~s}$ perisaccade period, except for an $\sim 100 \mathrm{~ms}$ period starting $\sim 100 \mathrm{~ms}$ after saccade onset.

We next examined the subset of cue position-selective neurons that also showed reward modulation in either cue position. Figure $2 C$ shows the population results in the same format as Figure $2 B$. Reward modulation in one cue position was concentrated in the first $500 \mathrm{~ms}$ after cue onset and in the perisaccade period in both FEF and caudate. It was absent in the late memory period for both regions. FEF showed a higher percentage of such reward modulation in the period beginning at $\sim 100 \mathrm{~ms}$ after cue onset and lasting intermittently for $\sim 130 \mathrm{~ms}$. In another period beginning shortly after saccade onset, caudate showed a higher percentage. To test whether the larger number of cue positionselective neurons in FEF accounted for these differences, we recomputed the percentage and confidence intervals with normalization by the number of cue position-selective neurons at each time bin. The normalized results are shown in Figure 2D. FEF continued to show a significantly higher percentage in the initial period after cue onset, but this was reversed soon, with caudate showing a higher percentage for $\sim 100 \mathrm{~ms}$ with occasional exceptions. The timing of the difference in reward modulation of saccade-related activity was not changed by the normalization procedure. Thus, cue position selectivity is more prevalent in FEF neurons. However, within the population of cue positionselective neurons, it is more likely to observe additional reward modulation in caudate.

In addition to the difference in the percentage of rewardmodulated neurons, we examined whether reward preference of these cells also displayed different patterns in FEF and caudate (Fig. $2 E$ ). We focused on three time periods: cue, presaccade, and postsaccade periods. We counted the numbers of neurons showing four types of modulation patterns: (1) RF-B type indicated larger activation in big-reward trials with preferred cue location (e.g., the FEF neuron in Figure 2A); (2) RF-S type indicated larger activation in small-reward trials with preferred cue location (e.g., the caudate neuron in Figure 2A); (3) non-B type indicated larger activation in big-reward trials with nonpreferred cue location; and (4) non-S type indicated larger activation in small-reward trials with nonpreferred cue location (e.g., postcue activity in the FEF neuron in Fig. $3 A$ ). Note that these four types of activation could occur at different times in the same cell. The frequency of
A
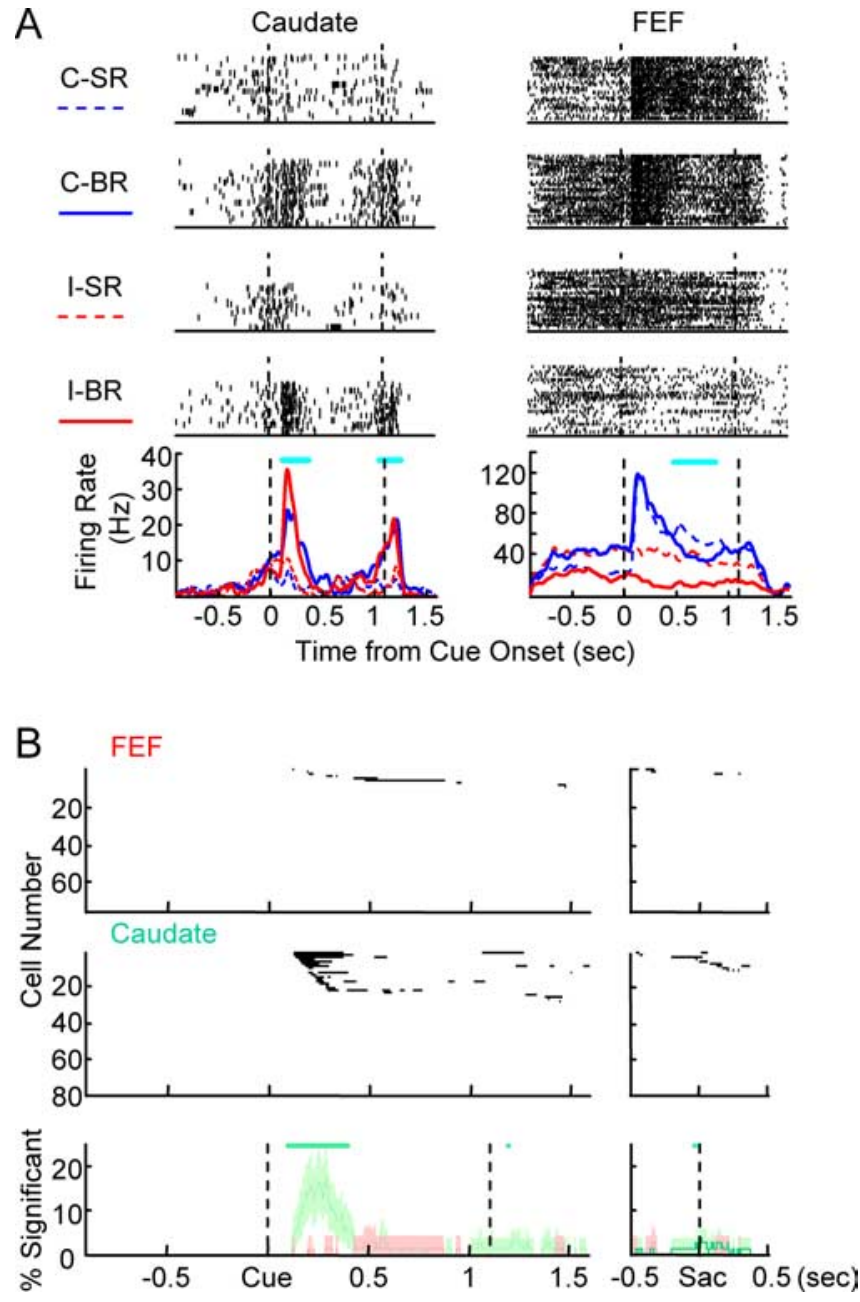

Figure 3. Reward size selectivity. A, Examples of neural activity showing reward size selectivity. The layout is the same as Figure $2 A$. Cyan bars indicate periods of significant reward size modulation. The FEF neuron was a rare example of reward size-selectivity. $\boldsymbol{B}$, Population data for reward size selectivity. The layout is the same as Figure $2 B$.

occurrence for each activation pattern was expressed as a percentage (count/total number of modulated neurons). As predicted from Figure $2 C$, a higher percentage of neurons showed modulation in the cue period than in the perisaccade period in both FEF and caudate. In caudate, the most prevalent type of modulation occurred with preferred cue location, with similar frequency for larger activation in big-reward and small-reward trials. In FEF, conversely, the most prevalent type of modulation was observed with nonpreferred cue locations, and the sign of modulation is such that there was larger activation in small-reward trials. This first impression, however, was not statistically significant $(p=$ 0.1783 and 0.2880 for the cue and postsaccade periods, respectively, $\chi^{2}$ test). Presaccade reward modulation was too infrequent in our sample for such a comparison.

To summarize, FEF neurons were significantly more likely to be cue position selective than caudate neurons throughout the task. In the subset of cue position-selective neurons, caudate neurons were more likely to also be reward modulated in cue response and saccade response. The reward modulation in FEF had an earlier onset, possibly attributable to earlier visual inputs. FEF neurons were rarely modulated by reward during the late memory period, consistent with previous results (Leon and Shadlen, 1999). In addition, caudate neurons also showed a lack of reward modulation in the same period. 


\section{Reward size selectivity is common in caudate but mostly lacking in FEF}

Examples of reward size-selective activity are shown in Figure $3 A$. The caudate neuron was more active in big-reward trials, regardless of cue position, in its visual response to the cue and saccaderelated activity around fixation disappearance. The FEF neuron was a rare example of reward size selectivity. Its response in the late memory period was weakly small reward selective. In contrast to this weak reward size selectivity, the same neuron showed clear reward position-selective activity before cue onset (for more details on reward position selectivity, see below, Reward position selectivity is present in both FEF and caudate) and contralateral cue position-selective cue response.

Figure $3 B$ shows the population results in the same format as Figure $2 \mathrm{~B}$. The population maps for FEF is almost devoid of significant time bins, whereas $\sim 30 \%$ of caudate neurons show significant reward size selectivity in the period after cue onset and smaller dark area in the perisaccade period. This impression was confirmed with the comparison between percentages of reward size-selective cells at each time bin. A significantly larger portion of caudate neurons showed reward size selectivity in the periods of $\sim 140-430 \mathrm{~ms}$ after cue onset and briefly after saccade onset. Reward size selectivity was mostly nonexistent in FEF.

\section{Reward position selectivity is present in both FEF and caudate}

Examples of reward position-selective activity are shown in Figure $4 A$. Note that, in the asymmetric reward task, big-reward position was varied in blocks. For example, contralateral bigreward blocks contained trials showing contralateral cue associated with big reward and trials showing ipsilateral cue associated with small reward. In other words, the first and fourth rows in the raster plots were data from one reward position condition (block), whereas the second and third rows were from the other reward position condition (block). The caudate neuron was highly active in both contralateral big-reward and ipsilateral small-reward trials although conspicuously silent in the other two trial types, indicating a strong selectivity for contralateral position as the big-reward position. This reward positionselective activity began $\sim 200 \mathrm{~ms}$ before cue onset and terminated $\sim 240 \mathrm{~ms}$ after cue onset. The FEF neuron also showed significant selectivity for contralateral position as the big-reward position in its precue activity. This reward position-selective activity began $\sim 600 \mathrm{~ms}$ before cue onset and continued until $\sim 170 \mathrm{~ms}$ after cue onset. Another example of reward position-selective activity can be seen in the FEF neuron shown in Figure $3 A$, in which contralateral big-reward position selective activity began $\sim 700 \mathrm{~ms}$ before cue onset and continued until $\sim 100 \mathrm{~ms}$ after cue onset.

Population results (Fig. $4 B$ ) show that FEF and caudate have similar percentage of cells with reward position selectivity for almost all time bins. This type of modulation usually began before cue onset and ended shortly after cue onset in both regions. The exact timing of the onset of this modulation was variable and not statistically different between FEF and caudate. The timing of the offset was more locked to the cue onset. There was a higher percentage in caudate at $\sim 130 \mathrm{~ms}$ after cue onset, a period corresponding to the average offset of reward position-selective activity. This difference lasted $\sim 14 \mathrm{~ms}$, suggesting that the reward position selectivity activity was terminated earlier in FEF than caudate by $\sim 14 \mathrm{~ms}$.

Although reward position modulation was present in similar frequency in FEF and caudate, there may be a difference in pre-
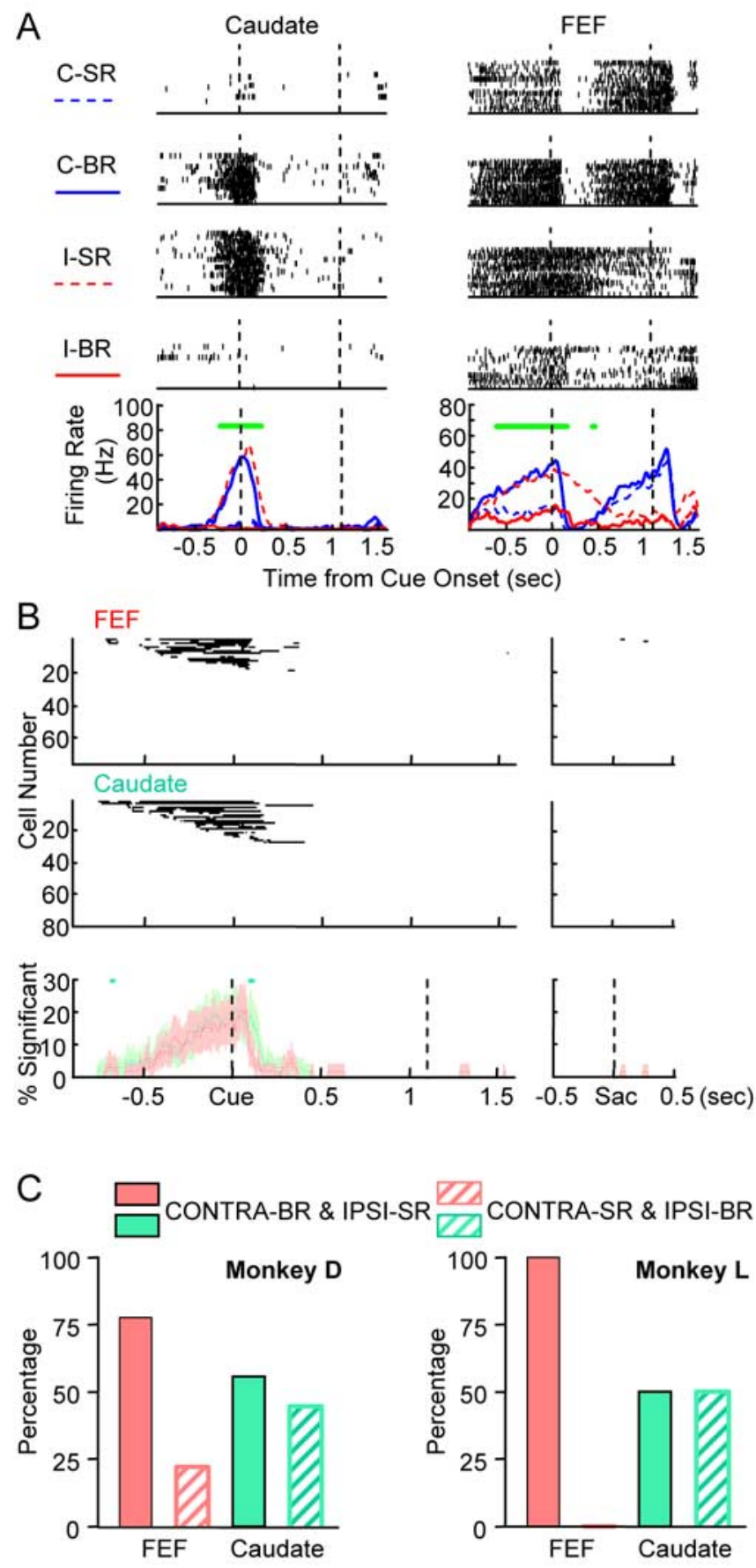

Figure 4. Reward position selectivity. $A$, Examples of neural activity showing reward position selectivity. The layout is the same as Figure $2 A$. Green bars indicate periods of significant reward position modulation. The FEF neuron also showed significant single condition selectivity after cue onset and cue position selectivity in late memory period and around saccade onset. $\boldsymbol{B}$, Population data for reward position selectivity. The layout is the same as Figure 2B.C, Reward position preference of reward position-selective neurons. FEF neurons showed contralateralbig reward dominance, whereas similar proportions of caudate neurons preferred the contralateral or ipsilateral target associated with big reward.

ferred big-reward positions. We grouped neurons that were tested using nonvertical cue positions and showed reward position-selective activity before the onset of early cue response. As shown in Figure $4 C$, the majority of FEF neurons preferred contralateral cues being associated with big reward. In contrast, similar proportion of caudate neurons showed big-reward posi- 

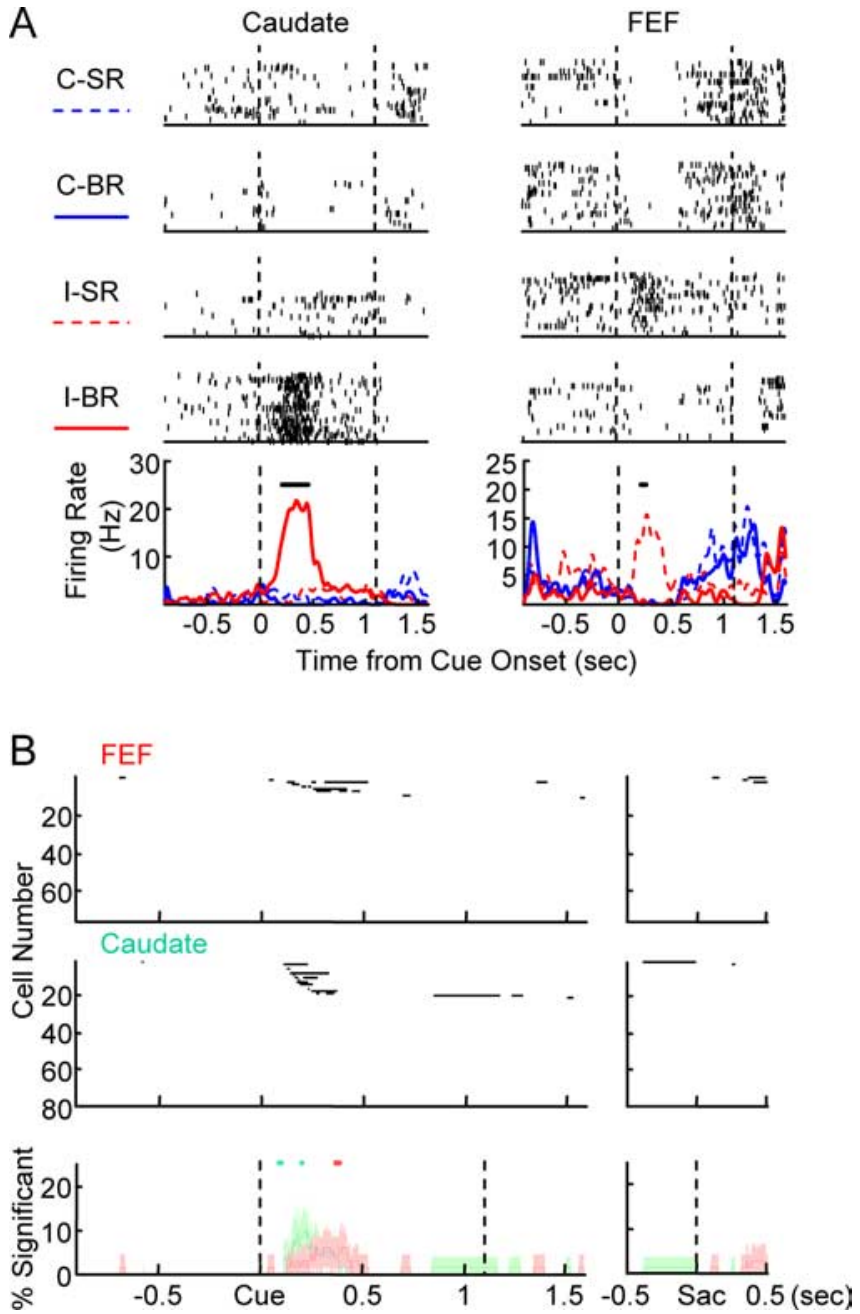

Figure 5. Single condition selectivity. $\boldsymbol{A}$, Examples of neural activity showing single condition selectivity. The layout is the same as Figure $2 A$. Black bars indicate periods of significant single condition selectivity. The FEF neuron also showed significant cue position selectivity in the late memory period and around saccade onset. $\boldsymbol{B}$, Population data for single condition selectivity. The layout is the same as Figure $2 B$.

tion preference to contralateral or ipsilateral. The laterality we observed here is weaker than reported previously in caudate (Takikawa et al., 2002), which may reflect sampling difference or individual variability of monkeys. Nonetheless, within the same monkeys in this study, the difference in laterality between FEF and caudate was statistically significant ( $p=0.0439, \chi^{2}$ test).

To summarize, reward position-selective activity is present in both FEF and caudate with similar frequency at time periods before cue onset. Such activity is terminated earlier in FEF than caudate. In FEF, the preferred reward position condition is dominantly contralateral cues with big reward, whereas in caudate, similar number of cells preferring contralateral cues associated with either big or small reward.

Single condition selectivity is present in both FEF and caudate Two main types of single condition-selective activity were observed. The first, more common, type of single conditionselective activity is shown in Figure $5 A$. The caudate neuron was active only in big-reward trials after ipsilateral cue onset. The FEF neuron was more active in the small-reward trials after ipsilateral cue onset than all of the other three types of trials.

Another type of single condition-selective activity can be seen
A All FEF Cells

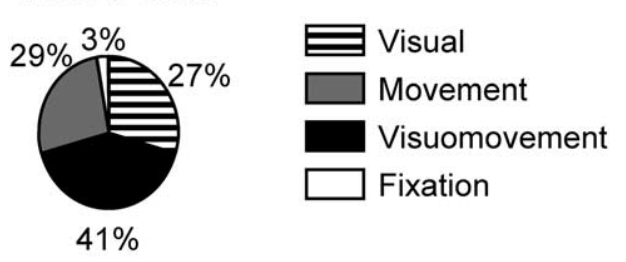

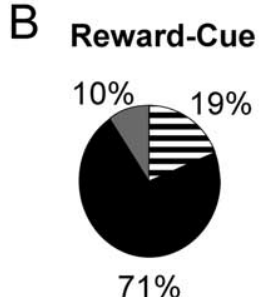

$71 \%$

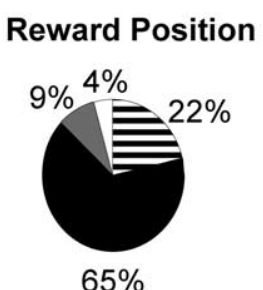

$65 \%$
Single Condition

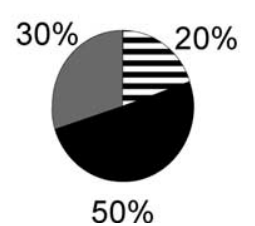

Figure 6. Distribution of reward modulation in traditional FEF neuron categories. $\boldsymbol{A}$, Percentage of neurons in the visual, visuomovement, movement, and fixation categories for all 75 FEF neurons. $\boldsymbol{B}$, Percentage of reward-modulated neurons in the four categories. The numbers of neurons showing each of the three types of reward modulation are 21,23 , and 10 , respectively. Visuomovement neurons in FEF dominate in every type of reward modulation. Note that "Reward-Cue" is an abbreviation for "reward modulation in one cue position."

in the two examples shown in Figure 4A. Both cells showed higher activity before cue onset in blocks in which contralateral cues were associated with big reward. After the cue onset, activity was reduced rapidly if the contralateral (big reward) cue appeared. Activity in trials with ipsilateral (small reward) cue lingered longer before eventually returning to baseline level. This resulted in periods when the two cells were active in only ipsilateral small-reward trials. In other words, they showed single condition selectivity after cue onset.

In the population, single condition-selective activity was observed almost exclusively in the early postcue period (Fig. 5B). During this period, 14 cells in caudate and six cells in FEF showed selectivity to a single condition not accounted for by the time course of reward position-selective activity, similar to the examples in Figure $5 A$. In three cells in caudate and two cells in FEF, reward position-selective activity lingered longer after presentation of cues associated with small reward, similar to the examples shown in Figure $4 \mathrm{~A}$. One cell in caudate showed nonspecific activity before cue onset, and this activity was terminated after cue onset more quickly in big-reward contralateral cue trials than in the other three types of trials. Overall, the percentage of single condition-selective neurons is comparable between FEF and caudate. More caudate neurons had earlier onset of this type of reward modulation than FEF neurons.

\section{Distribution of reward modulation in traditional FEF neuron categories}

In previous studies characterizing basic properties of FEF neurons, four categories have often been used: visual, visuomovement, movement, and fixation neurons (Bruce and Goldberg, 1985). It has also been elegantly shown that these four types of neurons have different descending projection patterns and receive different information from the SC-mediodorsal thalamusFEF ascending pathway (Segraves and Goldberg, 1987; Sommer and Wurtz, 2000, 2004). We next tested whether reward modulation in FEF is limited to specific neural categories. Based on visual inspection, the numbers of visual, visuomovement, movement, and fixation neurons are 22, 32, 19, and 1, respectively (Fig. 
Monkey D: FEF
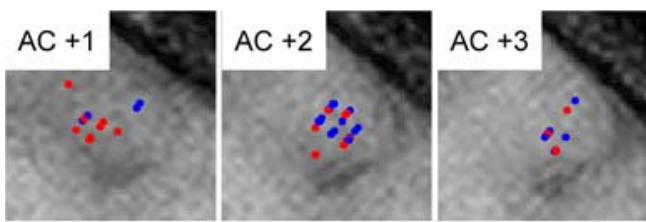

$5 \mathrm{~mm}$

Monkey D: caudate
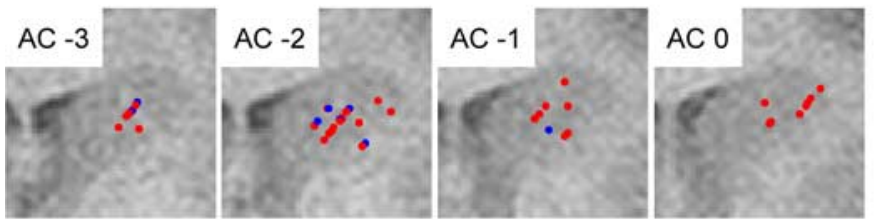

Monkey L: FEF
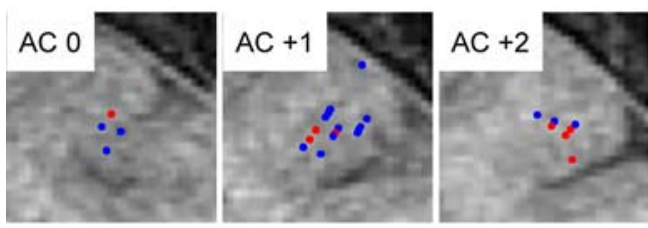

Monkey L: caudate
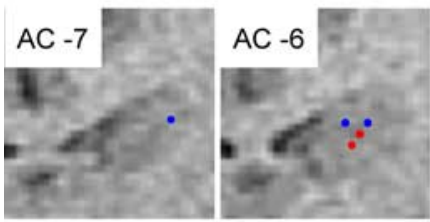

$5 \mathrm{~mm}$
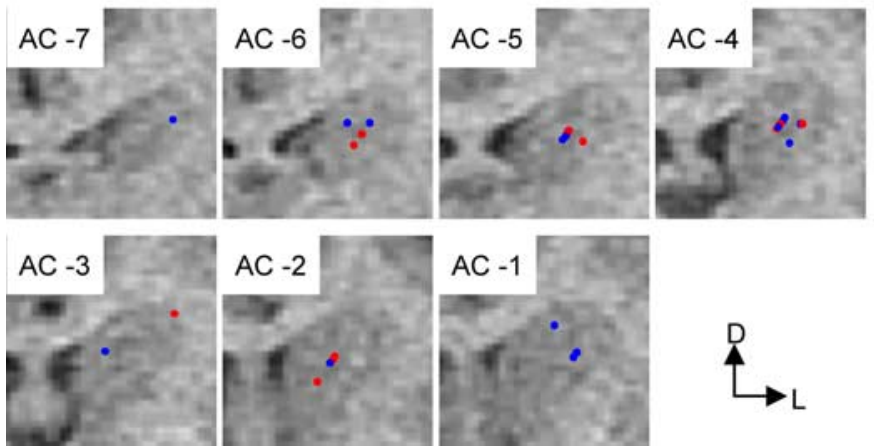

Figure 7. Locations of recorded neurons. Neuron localization is estimated based on the depth of the electrode at time of recording and overlaid on MR images in coronal sections. Rightmost panels show representative full sections, with the yellow rectangles outlining the regions magnified for the left panels. $A C$ indicates the level of the anterior commissure. Positive and negative numbers indicate the distance (in millimeters) anterior and posterior to $A C$, respectively. Red and blue dots indicate neurons with and without any of the four types of reward modulation, respectively. D, Dorsal; L, lateral.

$6 \mathrm{~A})$. One neuron did not fit into any of the conventional categories. Because little reward modulation was observed in the perisaccade activity, presaccadic and postsaccadic neurons were lumped into the movement category. This category includes 10 presaccadic neurons, seven postsaccadic neurons, and two neurons with both presaccadic and postsaccadic activity related to saccades in opposite directions.

As shown in Figure $6 B$, all three types of reward modulation were represented in visual, visuomovement, and movement neurons. The most salient feature is that most reward-modulated neurons belong to the visuomovement category. For statistical analysis, we excluded fixation neurons because of their small number. The dominance of visuomovement neurons is significant for reward modulation in one cue position and for reward position modulation ( $p=0.0387$ and 0.0498 , respectively, $\chi^{2}$ test). We also examined whether the proportion of rewardmodulated neurons is different between neuron categories. We found that, for reward modulation in one cue position, significantly larger proportion of visuomovement neurons is modulated than visual or saccade neurons ( $p=0.0239$ and 0.0045 ,
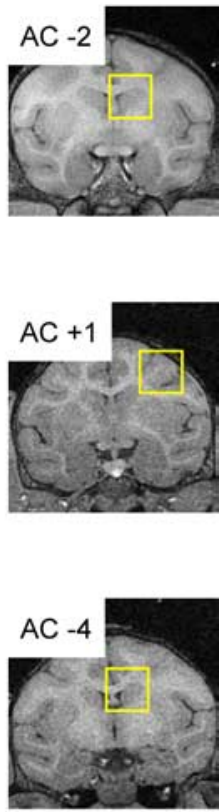

respectively, $\chi^{2}$ test). For reward position modulation, a significantly larger proportion of visuomovement neurons is modulated than saccade neurons ( $p=0.0045$, $\chi^{2}$ test), whereas the difference between visuomovement and visual neurons did not reach significance criterion $(p=$ $0.0576, \chi^{2}$ test). To summarize, the majority of reward-modulated neurons belong to the visuomovement category. The cue position-selective activity is more likely to also be reward modulated in visuomovement than visual or saccade neurons. Reward position modulation is more prevalent in visuomovement neurons than in saccade neurons.

\section{Locations of recorded neurons}

Locations of recorded neurons were reconstructed and overlaid on MR images in coronal sections (Fig. 7). Red dots represent cells showing any one of the four types of reward modulation. No distinct pattern was found in caudate for distribution of reward-modulated neurons. In FEF of monkey $\mathrm{D}$, reward-modulated neurons were more concentrated away from the surface of the gyrus ( $p=0.0081$, MannWhitney test). Although there is a hint of similar pattern in monkey L, it was not statistically significant.

\section{Discussion}

Using an asymmetrically rewarded memory-guided saccade task, we found that many neurons in FEF showed spatially selective reward modulation, similar to that observed in caudate. FEF neurons rarely showed pure reward size modulation, in clear contrast to caudate neurons.

More specifically, FEF has a higher percentage of cue position-selective cells throughout the task period after cue onset. This difference is especially prominent during the period immediately after cue onset. Within the population of cue position-selective cells, an FEF neuron is less likely to also be modulated by reward than a caudate neuron. Consistent with previous results, FEF neurons were not modulated by reward in the memory period (Leon and Shadlen, 1999). This is also true for caudate neurons. The percentage of FEF neurons showing reward modulation in visual response seemed higher than that observed previously (Roesch and Olson, 2003). This may be attributable to the difference in the behavioral tasks used. In our experiment, the position of a cue determines the size of the upcoming reward, whereas in the previous experiment, the size of the upcoming reward was signaled before the cue position. The coincidence of both spatial and reward information might amplify the magnitude of reward modulation of visual responses.

Both FEF and caudate contain similar proportion of reward position-selective neurons. The termination of reward positionselective activity tends to be earlier in FEF than caudate. Most neurons in FEF prefer the contralateral cue-big reward association to ipsilateral cue-big reward association. Such laterality is 
much weaker in caudate. The reward position-selective activity may seem similar to the anticipatory activity observed previously in a free-choice task (Coe et al., 2002). Such comparison, however, should be cautioned, because the nature of the anticipatory activity may be different. In a forced-choice task like ours, the anticipatory activity may reflect the preference of the big-reward position but not necessarily the final saccade decision, whereas in the free-choice task, it is likely that saccade decision was formed before target appearance and could be reflected in the anticipatory activity.

Given the direct anatomical projection from FEF to caudate, it is natural to hypothesize that the reward information represented in caudate is at least partly derived from the FEF inputs. This hypothesis is consistent with the observation of a substantial number of FEF neurons showing reward modulation in one cue position and the earlier onset of such modulation in FEF than caudate. It is further supported by the observation of a similar time course of reward position-selective activity in FEF and caudate. However, because the FEF-caudate projection is primarily ipsilateral (Stanton et al., 1988; Saint-Cyr et al., 1990; Shook et al., 1991; Parthasarathy et al., 1992), the substantial representation of both contralateral and ipsilateral big-reward positions in caudate suggests additional sources of reward position modulation. Candidate cortical areas include prefrontal cortex, supplementary eye fields, and orbitofrontal cortex, all of which participate in saccade generation and have bilateral projections to caudate (Selemon and Goldman-Rakic, 1985; Saint-Cyr et al., 1990; Shook et al., 1991; Parthasarathy et al., 1992; Eblen and Graybiel, 1995). Supplementary eye fields, in particular, have been shown to show reward position-selective activity in a free-choice task (Coe et al., 2002).

Although our results are consistent with a flow of reward information from FEF to caudate, it remains to be tested, for example, using antidromic activation techniques, whether such reward information is indeed present in the caudate-projecting neurons in FEF. Alternatively, a "reverse" flow of information is conceivable from the basal ganglia to FEF by way of the ascending pathway through the superior colliculus and/or thalamus. A third alternative would be that reward modulation, such as the reward position selectivity, is a network phenomenon that is generated and maintained by the whole corticobasal ganglia-cortical loop. If so, disruption in any structure may eliminate such modulation in the whole loop. These alternatives should be explored in future to pinpoint the sources of reward modulation in caudate.

One thing is clear from our data: the lack of reward sizeselective activity in FEF strongly suggests that such activity in caudate does not come from FEF. Given our current knowledge of neural activity in various brain regions in tasks similar to ours, the most promising candidate for the origin of such caudate activity is the substantia nigra pars compacta $(\mathrm{SNc})$, which shows nonspatial reward modulation (Schultz, 1998; Kawagoe et al., 2004). Other candidate regions include the lateral prefrontal cortex (Kobayashi et al., 2002; Wallis and Miller, 2003), the orbitofrontal cortex (Tremblay and Schultz, 1999; Wallis and Miller, 2003; Roesch and Olson, 2004), and the premotor area caudal to the arcuate sulcus, which has been shown to have high degree of reward modulation and a lesser degree of directional selectivity in a variable reward task (Roesch and Olson, 2003).

To consider our results in the context of saccade generation, we summarized, in Figure 8, our results and the previous results from other areas using similar asymmetrically rewarded memory-guided saccade tasks (Sato and Hikosaka, 2002; Takikawa et al., 2002; Ikeda and Hikosaka, 2003; Kawagoe et al.,

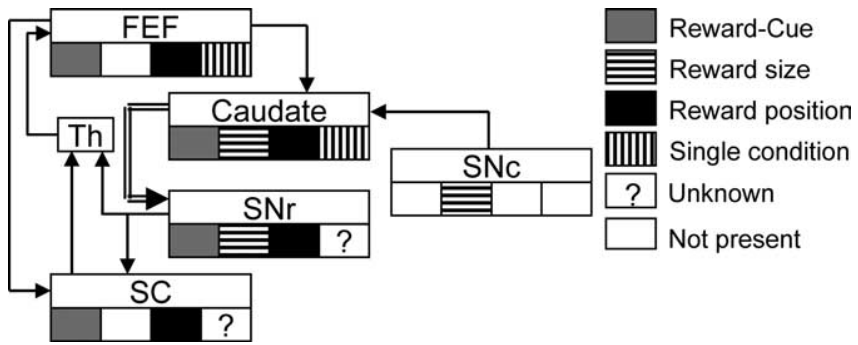

Figure 8. Illustrative summary of reward modulation in various saccade-related regions. Results from this and previous reports using similar asymmetrically rewarded saccade tasks are shown. The projections from caudate to SNr (double-lined arrow) include both the direct and indirect pathways. Note that "Reward-Cue" is an abbreviation for "reward modulation in one cue position." Th, Thalamus.

2004). Single condition-selective activity has not been specifically studied in other areas. However, given the extensive results on spatially nonselective nature of dopamine neurons in SNc, we assumed that single condition-selective activity is not present there. Pure reward size-selective activity was common in SNr but not present in SC (O. Hikosaka, T. Ikeda, and M. Sato, unpublished data). Even with a crude summary as such, it is clear that pure reward size-selective, nonspatial reward modulation in caudate is neither present in FEF nor transmitted downstream to SC. In contrast, spatially selective reward modulation, be it reward modulation in one cue position or reward position selectivity, is not only present in FEF and caudate but also transmitted downstream to SC. These results are consistent with FEF and SC being more intimately related to generation of saccades with specific directions. They also support additional reward modulation of cognitive functions of caudate. Because of the close link between reward manipulation and attentional modulation in our experimental paradigm (Maunsell, 2004), the detailed nature of the spatially selective reward modulation awaits additional investigation.

The asymmetric reward paradigm revealed activation patterns in FEF neurons that were not predicted from data using conventional tasks with equal reward (e.g., the prolonged activation after ipsilateral cue onset in small-reward trials in Fig. $4 \mathrm{~A}$, or the activation specifically after ipsilateral cue onset in small-reward trials in Fig. 5A). This suggests that FEF activity can be highly context dependent and that FEF may have flexible roles in the process of sensorimotor transformation. In this process, it is generally thought that visuomovement neurons represent an important stage. We found that most reward-modulated neurons belong to the visuomovement category. This suggests that visuomovement neurons may also be a key stage in which motivational information is integrated for the sensorimotor transformation.

Interestingly, caudate contains a higher percentage of rewardmodulated neurons than FEF in the postsaccadic period, even without normalization to the number of cue position-selective neurons. This suggests that, in addition to evaluating the cuereward association, caudate may also contribute to evaluation of the saccade-reward contingency. Such a function may be especially important during the initial learning of the task, while the association among cue position, saccade, and reward is established.

In conclusion, spatially selective reward information is present in both FEF and caudate, making it likely that both structures contribute to the behavioral reward bias. Pure reward modulation, conversely, may serve as an intermediary in the reward expectation to behavioral bias transformation but might not directly contribute to the behavioral bias. 


\section{References}

Bruce CJ, Goldberg ME (1985) Primate frontal eye fields. I. Single neurons discharging before saccades. J Neurophysiol 53:603-635.

Coe B, Tomihara K, Matsuzawa M, Hikosaka O (2002) Visual and anticipatory bias in three cortical eye fields of the monkey during an adaptive decision-making task. J Neurosci 22:5081-5090.

Eblen F, Graybiel AM (1995) Highly restricted origin of prefrontal cortical inputs to striosomes in the macaque monkey. J Neurosci 15:5999-6013.

Huerta MF, Krubitzer LA, Kaas JH (1986) Frontal eye field as defined by intracortical microstimulation in squirrel monkeys, owl monkeys, and macaque monkeys. I. Subcortical connections. J Comp Neurol 253:415-439.

Ikeda T, Hikosaka O (2003) Reward-dependent gain and bias of visual responses in primate superior colliculus. Neuron 39:693-700.

Judge SJ, Richmond BJ, Chu FC (1980) Implantation of magnetic search coils for measurement of eye position: an improved method. Vision Res 20:535-538.

Kawagoe R, Takikawa Y, Hikosaka O (1998) Expectation of reward modulates cognitive signals in the basal ganglia. Nat Neurosci 1:411-416.

Kawagoe R, Takikawa Y, Hikosaka O (2004) Reward-predicting activity of dopamine and caudate neurons-a possible mechanism of motivational control of saccadic eye movement. J Neurophysiol 91:1013-1024.

Kobayashi S, Lauwereyns J, Koizumi M, Sakagami M, Hikosaka O (2002) Influence of reward expectation on visuospatial processing in macaque lateral prefrontal cortex. J Neurophysiol 87:1488-1498.

Lauwereyns J, Watanabe K, Coe B, Hikosaka O (2002a) A neural correlate of response bias in monkey caudate nucleus. Nature 418:413-417.

Lauwereyns J, Takikawa Y, Kawagoe R, Kobayashi S, Koizumi M, Coe B, Sakagami M, Hikosaka O (2002b) Feature-based anticipation of cues that predict reward in monkey caudate nucleus. Neuron 33:463-473.

Leon MI, Shadlen MN (1999) Effect of expected reward magnitude on the response of neurons in the dorsolateral prefrontal cortex of the macaque. Neuron 24:415-425.

Maunsell JH (2004) Neuronal representations of cognitive state: reward or attention? Trends Cogn Sci 8:261-265.

Parthasarathy HB, Schall JD, Graybiel AM (1992) Distributed but convergent ordering of corticostriatal projections: analysis of the frontal eye field and the supplementary eye field in the macaque monkey. J Neurosci 12:4468-4488.

Roesch MR, Olson CR (2003) Impact of expected reward on neuronal activity in prefrontal cortex, frontal and supplementary eye fields and premotor cortex. J Neurophysiol 90:1766-1789.

Roesch MR, Olson CR (2004) Neuronal activity related to reward value and motivation in primate frontal cortex. Science 304:307-310.

Saint-Cyr JA, Ungerleider LG, Desimone R (1990) Organization of visual cortical inputs to the striatum and subsequent outputs to the pallidonigral complex in the monkey. J Comp Neurol 298:129-156.

Sato M, Hikosaka O (2002) Role of primate substantia nigra pars reticulata in reward-oriented saccadic eye movement. J Neurosci 22:2363-2373.

Schultz W (1998) Predictive reward signal of dopamine neurons. J Neurophysiol 80:1-27.

Segraves MA, Goldberg ME (1987) Functional properties of corticotectal neurons in the monkey's frontal eye field. J Neurophysiol 58:1387-1419.

Selemon LD, Goldman-Rakic PS (1985) Longitudinal topography and interdigitation of corticostriatal projections in the rhesus monkey. J Neurosci 5:776-794.

Shook BL, Schlag-Rey M, Schlag J (1991) Primate supplementary eye field. II. Comparative aspects of connections with the thalamus, corpus striatum, and related forebrain nuclei. J Comp Neurol 307:562-583.

Sommer MA, Wurtz RH (2000) Composition and topographic organization of signals sent from the frontal eye field to the superior colliculus. J Neurophysiol 83:1979-2001.

Sommer MA, Wurtz RH (2004) What the brain stem tells the frontal cortex. I. Oculomotor signals sent from superior colliculus to frontal eye field via mediodorsal thalamus. J Neurophysiol 91:1381-1402.

Stanton GB, Goldberg ME, Bruce CJ (1988) Frontal eye field efferents in the macaque monkey. I. Subcortical pathways and topography of striatal and thalamic terminal fields. J Comp Neurol 271:473-492.

Takikawa Y, Kawagoe R, Hikosaka O (2002) Reward-dependent spatial selectivity of anticipatory activity in monkey caudate neurons. J Neurophysiol 87:508-515.

Tremblay L, Schultz W (1999) Relative reward preference in primate orbitofrontal cortex. Nature 398:704-708.

Wallis JD, Miller EK (2003) Neuronal activity in primate dorsolateral and orbital prefrontal cortex during performance of a reward preference task. Eur J Neurosci 18:2069-2081. 